\title{
On a Holomorphic Fiber Bundle with Meromorphic Structure
}

\author{
By
}

\section{Akira FuJIKI*}

\section{Introduction}

Let $f: X \rightarrow Y$ be a proper surjective morphism of compact complex manifolds. Let $U \subseteq Y$ be a Zariski open subset over which $f$ is smooth. Let $X_{U}=f^{-1}(U)$ and let $f_{U}: X_{U} \rightarrow U$ be the induced morphism. Assume that $f_{U}$ is a holomorphic fiber bundle with typical fiber $F$ and the structure group $H$. Let $G_{U} \rightarrow U$ be the holomorphic fiber bundle associated with $f_{U}$ with typical fiber $H$ with the adjoint action of $H$ on itself so that $G_{U}$ acts naturally on $X_{U}$ over $U$. Let $I_{U} \rightarrow U$ be the principal $H$-bundle associated to $f_{U}$. Then $G_{U}$ acts naturally on $I_{U}$ over $U$ also. We say that $f_{U}$ is a holomorphic fiber bundle with meromorphic structure if there exists a compact complex space $G^{*}$ (resp. $I^{*}$ ) over $Y$ containing $G_{U}$ (resp. $I_{U}$ ) as a Zariski open subset such that the action of $G_{U}$ on $X_{U}$ (resp. $I_{U}$ ) extends 'meromorphically' to that of $G^{*}$ on $X$ (resp. $I^{*}$ ). Then in this paper we shall prove the following: Suppose that $f_{U}$ is a holomorphic fiber bundle with meromorphic structure for some $G^{*}$ and $I^{*}$ as above. Then

1) there exists a 'generic quotients' $X / G^{*}$ of $X$ by $G^{*}$ over $Y$, and

2) $X / G^{*}$ is bimeromorphic to the product space $(F / H) \times Y$ where $F / H$ is a generic quotient of $F$ by $H .{ }^{1}$

Actually in this paper, these results are obtained in a more general setting of comparing two proper morphisms $f_{i}: X_{\imath} \rightarrow Y, i=1$, 2, over $Y$ having isomorphic general fibers (cf. Theorems 1 and 2 ); the above special case corresponds to the case where one of the $f_{i}$ is isomorphic to the projection $p: F \times Y \rightarrow Y$. (This generalization is in a sense parallel with Grothendieck's generalization [7] of the theory of fiber bundles to the theory of general fiber spaces with structure sheaf.)

Section 1 is preliminary, and in Section 2 we prove Theorems 1 and 2 mentioned above. Then in Section 3 we shall give some general examples which appear naturally in the study of the structure of compact complex manifolds in $\mathcal{C}[5]$; indeed, the application to these examples is the principal motivation for this paper. Finally in Section 4, as a reference for [5], we gather some results

Communicated by S. Nakano, January 21, 1982.

* Yoshida College, Kyoto University, Kyoto 606, Japan.

1) The assumption on $I_{U}$ is unnecessary for the assertion 1 ). 
related to the subject of this paper.

In this paper a complex variety means a reduced and irreducible complex space. Let $f: X \rightarrow Y$ be a proper surjective morphism of complex varieties. Then we write $f \in \mathcal{C} / Y$ if there exist a proper Kähler morphism $g: Z \rightarrow Y$ (cf. [4]) and a surjective meromorphic $Y$-map $\phi: Z \rightarrow X$.

\section{$\S 1$. Preliminaries and Basic Definitions}

1.1. a) Let $Y$ be a complex space. Then a relative complex Lie group over $Y$ is a complex space $G$ over $Y$ with a holomorphic section $e: Y \rightarrow G$ (the identity section) and $Y$-morphisms $\mu=\mu_{G / Y}: G \times{ }_{Y} G \rightarrow G$, and $\iota=\iota_{X / Y}: G \rightarrow G$ (relative group multiplication and inversion) satisfying the usual axioms of group law (cf. [11], Def. 0.1). Then a relative complex Lie subgroup of $G$ is a complex subspace $H$ of $G$ which itself is a relative complex Lie group over $Y$ with respect to the 'restrictions' of $e, \mu$ and $\iota$ to $H$. Let $f: X \rightarrow Y$ be a morphism of complex spaces and $G$ a relative complex Lie group over $Y$. Then a relative (biholomorphic) action of $G$ on $X$ over $Y$ is a $Y$-morphism $\sigma: G \times_{Y} X \rightarrow X$ satisfying the usual axioms of operation (cf. [11], Def. 0.3).

b) Let $f: X \rightarrow Y$ and $f^{\prime}: X^{\prime} \rightarrow Y$ be proper surjective flat morphisms of complex spaces. Let $(\mathrm{An} / Y)$ be the category of complex spaces over $Y$. Then we define the contravariant functor $\operatorname{Isom}_{Y}\left(X, X^{\prime}\right):(\mathrm{An} / Y) \rightarrow($ Sets $)$ by the following formula; $\operatorname{Isom}_{Y}\left(X, X^{\prime}\right)(\tilde{Y}):=$ the set of $\tilde{Y}$-isomorphisms $\varphi: X \times_{Y} \tilde{Y} \rightarrow X^{\prime} \times_{Y} \tilde{Y}$ where $\tilde{Y} \in(\mathrm{An} / Y)$. Let $D_{X \times_{X X^{\prime} / Y} \rightarrow Y}$ be the relative Douady space associated to the morphism $f \times_{Y} f^{\prime}: X \times_{Y} X^{\prime} \rightarrow Y$. Then $\operatorname{Isom}_{Y}\left(X, X^{\prime}\right)$ is represented by a Zariski open subset $\operatorname{Isom}_{Y}\left(X, X^{\prime}\right)$ of $D_{X_{\times} X^{\prime} / Y}$ (cf. Schuster [13]). We set $\operatorname{Aut}_{Y} X:=\operatorname{Isom}_{Y}(X, X)$. Then $\operatorname{Aut}_{Y} X$ has the natural structure of a complex Lie group over $Y$, acting naturally on $X$ over $Y$.

When $Y$ is a point, we write $\operatorname{Isom}\left(X, X^{\prime}\right)$ and Aut $X$ instead of $\operatorname{Isom}_{Y}\left(X, X^{\prime}\right)$ and $\operatorname{Aut}_{Y} X$ respectively. Aut $X$ is thus the automorphism group of $X$ as a complex Lie group in the usual sense.

For any $\tilde{Y} \in(\mathrm{An} / Y)$ we have the natural isomorphisms $\operatorname{Isom}_{Y}\left(X, X^{\prime}\right) \times_{Y} \tilde{Y}$ $\cong \operatorname{Isom}_{\tilde{Y}}\left(\tilde{X}, \tilde{X}^{\prime}\right)$ and $\left(\operatorname{Aut}_{Y} X\right) \times_{Y} \tilde{Y} \cong \operatorname{Aut}_{\tilde{Y}} \tilde{X}$ where $\tilde{X}=X \times_{Y} \tilde{Y}$ and $\tilde{X}^{\prime}=X^{\prime} \times_{Y} \tilde{Y}$ (cf. [8], [13]). In particular we have for each $y \in Y, \operatorname{Isom}_{Y}\left(X, X^{\prime}\right)_{y} \cong \operatorname{Isom}\left(X_{y}, X_{y}^{\prime}\right)$ and $\left(\operatorname{Aut}_{Y} X\right)_{y} \cong$ Aut $X_{y}$.

c) $\operatorname{Aut}_{Y} X$ and $\operatorname{Aut}_{Y} X^{\prime}$ act naturally on $\operatorname{Isom}_{Y}\left(X, X^{\prime}\right)$ over $Y$ (from the right in the case of $\left.\operatorname{Aut}_{Y} X\right)$. In relation to these actions we shall define the notion of principai subspace of $\operatorname{Isom}_{Y}\left(X, X^{\prime}\right)$ in a rather primitive way.

i) When $Y$ is a point, then with respect to this action Isom $\left(X, X^{\prime}\right)$ becomes a principal homogeneous space under either of Aut $X$ and Aut $X^{\prime}$, i. e., for any $h \in \operatorname{Isom}\left(X, X^{\prime}\right)$ the induced maps $\sigma_{h}$ : Aut $X \rightarrow \operatorname{Isom}\left(X, X^{\prime}\right), \sigma_{h}(g)=h g$, and $\sigma_{h}^{\prime}:$ Aut $X^{\prime} \rightarrow \operatorname{Isom}\left(X, X^{\prime}\right), \quad \sigma_{h}^{\prime}\left(g^{\prime}\right)=g^{\prime} h$, are isomorphic where $g \in$ Aut $X$ and $g^{\prime} \in$ Aut $X^{\prime}$. We shall call any isomorphism Aut $X \rightarrow \operatorname{Isom}\left(X, X^{\prime}\right)$ obtained in 
this way admissible. The composition $h_{*}:=\sigma_{h}^{\prime-1} \sigma_{h}$ : Aut $X \rightarrow$ Aut $X^{\prime}$ is an isomorphism of complex Lie groups, and is given by $h_{*}(g)=h g h^{-1}, g \in$ Aut $X$. Hence $h(g x)=h_{*}(g) h(x)$ for any $g \in$ Aut $X$ and $x \in X$. Now let $I \leqq$ Isom $X$ be a subspace. Then $I$ is called principal if there exist complex Lie subgroups $G \cong$ Aut $X$ and $G^{\prime} \cong$ Aut $X^{\prime}$ such that $G \cong I$ and $G^{\prime} \cong I$ under some admissible isomorphisms. In this case $G$ and $G^{\prime}$ are said to be associated to $I$.

ii) In the general case let $I \cong \operatorname{Isom}_{Y}\left(X, X^{\prime}\right)$ be any analytic subset. Assume that $X$ and $Y$ are varieties. Then $I$ is called principal if there exist relative complex Lie subgroups $G \subseteq$ Aut $_{Y} X$ and $G^{\prime} \cong$ Aut $_{Y} X^{\prime}$ such that for each $y \in Y, I_{y}$ is principal with the associated subgroups $G_{y} \subseteq$ Aut $X_{y}$ and $G_{y}^{\prime} \subseteq$ Aut $X_{y}$. In this case we call $G$ and $G^{\prime}$ associated to $I$.

1.2. a) We use the following terminology. Let $h: Z \rightarrow Y$ be a proper morphism of complex varieties and $V \leqq Y$ a Zariski open subset. Let $A \subseteq h^{-1}(V)$ be an analytic subset whose closure $\bar{A}$ in $Z$ is analytic. Then the essential closure $A^{*}$ of $A$ in $Z$ (over $Y$ ) is the union of those irreducible components of $\bar{A}$ which are mapped surjectively onto $Y$. Clearly, if $V^{\prime} \leqq V$ is another Zariski open subset, then the essential closure of $A \cap h^{-1}\left(V^{\prime}\right)$ in $Z$ coincides with $A^{*}$. Moreover, if $\bar{A}$ is proper over $Y$, there exists a Zariski open subset $U \subseteq Y$ such that for any $y \in U, A_{y} \subseteq A_{y}^{*}$ and $A_{y}^{*}$ is the closure of $A_{y}$. In fact, since $A^{*}$ is the closure of $A \cap A^{*}$, it suffices to show the assertion with $A^{*}$ replaced by $\bar{A}$. In this case the proof is standard.

b) Let $f: X \rightarrow Y$ and $f^{\prime}: X^{\prime} \rightarrow Y$ be proper surjective morphisms of complex varieties (not necessarily flat). Let $U \subseteq Y$ be a Zariski open subset over which both $f$ and $f^{\prime}$ are flat [1]. Then $\operatorname{Isom}_{U}\left(X_{U}, X_{U}^{\prime}\right)$ is Zariski open in $D_{X_{\times_{Y} X^{\prime} / Y}}$ $\supseteqq D_{X_{U \times U} X_{U}^{\prime Y}}$

Definition 1. $\operatorname{Isom}_{Y}^{*}\left(X, X^{\prime}\right)$ is the essential closure of $\operatorname{Isom}_{U}\left(X_{U}, X_{U}^{\prime}\right)$ in $D_{X \times_{Y} X^{\prime} / Y}$ over $Y$. We set $\operatorname{Aut}_{Y}^{*} X:=\operatorname{Isom}_{Y}^{*}(X, X)$. When $Y$ is a point, we simply write $\operatorname{Isom}^{*}\left(X, X^{\prime}\right)$ and Aut* $X$.

Remark 1. 1) $\operatorname{Isom}_{Y}^{*}\left(X, X^{\prime}\right)$ and Aut $X$ is independent of the choice of $U$ as above and depends only on $f$ and $f^{\prime}$ (cf. a)).

2) Let $\varphi: X_{U} \rightarrow X_{U}^{\prime}$ be a $Y$-isomorphism represented by a unique holomorphic section $s: U \rightarrow \operatorname{Isom}_{U}\left(X_{U}, X_{U}^{\prime}\right)$. Then $\varphi$ extends to a bimeromorphic $Y$-map $\varphi^{*}: X \rightarrow X^{\prime}$ if and only if $s$ extends to a meromorphic section $s^{*}: Y \rightarrow \operatorname{Isom}_{Y}^{*}\left(X, X^{\prime}\right)$.

3) The relative group multiplication $\mu_{U}: \operatorname{Aut}_{U} X_{U} \times_{U} \mathrm{Aut}_{U} X_{U} \rightarrow \mathrm{Aut}_{U} X_{U}$ and inversion $\iota_{U}: \operatorname{Aut}_{U} X_{U} \rightarrow \operatorname{Aut}_{U} X_{U}$ of relative complex Lie groups $\operatorname{Aut}_{U} X_{U}$ over $U$, and the natural relative action $\sigma_{U}$ : Aut $_{U} X_{U} \times_{U} X_{U} \rightarrow X_{U}$ of $\operatorname{Aut}_{U} X_{U}$ on $X_{U}$ over $U$ extend to meromorphic maps $\mu^{*}$ : Aut $X \times_{Y}^{*}$ Aut $_{Y}^{*} X \rightarrow \operatorname{Aut}_{Y}^{*} X, \iota^{*}:$ Aut $_{Y}^{*} X \rightarrow \operatorname{Aut}_{Y}^{*} X$ and $\sigma^{*}:$ Aut $_{Y}^{*} X \times_{Y} X \rightarrow X$ respectively. Moreover the identity section $e_{U}: U \rightarrow \operatorname{Aut}_{U} X_{U}$ extends to a meromorphic section $e^{*}: Y \rightarrow$ Aut $X$.

4) Let $\nu: \tilde{Y} \rightarrow Y$ be any proper surjective morphism of complex varieties. Set $\tilde{X}=X \times_{Y} \tilde{Y}$ and $\tilde{X}^{\prime}=X^{\prime} \times_{Y} \tilde{Y}$. Then we have the natural isomorphisms 
$\operatorname{Isom}_{Y}^{*}\left(X, X^{\prime}\right) \times_{Y} \tilde{Y} \cong \operatorname{Isom}_{\tilde{Y}}^{*}\left(\tilde{X}, \tilde{X}^{\prime}\right)$ and $\operatorname{Aut}_{Y}^{*} X \times_{Y} \tilde{Y} \cong \operatorname{Aut}_{\tilde{Y}}^{*} \tilde{X}$.

5) Suppose that $f, f^{\prime} \in \mathcal{C} / Y$. Then for any relatively compact open subset $V \leqq Y$ any irreducible component of $\operatorname{Isom}_{V}^{*}\left(X_{V}, X_{V}^{\prime}\right)$ and $\operatorname{Aut}_{V}^{*} X_{V}$ is proper over $Y$. This follows from [4].

1.3. a) Let $f: X \rightarrow Y$ be a proper morphism of complex varieties.

Definition 2. Let $G^{*} \subseteq$ Aut $X$ be an analytic subset such that any irreducible component of $G^{*}$ is mapped surjectively onto $Y$. Then we call $G^{*}$ (by abuse of language) a relative quasi-meromorphic (Lie) subgroup of Aut $X$ if there exists a Zariski open subset $U \subseteq Y$ such that $f$ is flat over $U$ and that $G_{U}$ $:=G^{*} \cap \mathrm{Aut}_{U} X_{U}$ is dense in $G^{*}$ and is a relative Lie subgroup of $\operatorname{Aut}_{U} X_{U}$ over $U$. If, further, $G^{*}$ is proper over $Y$, we call $G^{*}$ a relative meromorphic (Lie) subgroup of Aut $X$.

Remark 2. 1) If $Y$ reduces to a point, $G^{*}$, or more properly, $G=G^{*} \cap$ Aut $X$, is called a (quasi-)meromorphic subgroup of Aut*X (cf. [3]).

2) If $G^{*}$ is a relative quasi-meromorphic subgroup and $G_{U}$ is as above, then the relative group law $G_{U} \times{ }_{U} G_{U} \rightarrow G_{U}, G_{U} \rightarrow G_{U}$ (cf. 1.1) and the relative action $\sigma_{U}: G_{U} \times_{U} X_{U} \rightarrow X_{U}$ extend to meromorphic $Y$-maps $G^{*} \times{ }_{Y} G^{*} \rightarrow G^{*}, G^{*} \rightarrow G^{*}$ and $\sigma^{*}: G^{*} \times_{Y} X \rightarrow X$ respectively. Moreover the identity section $e_{U}: U \rightarrow G_{U}$ extends to a meromorphic section $e^{*}: Y \rightarrow G^{*}$. This follows from Remark 1,3).

b) Let $f: X \rightarrow Y$ and $f^{\prime}: X^{\prime} \rightarrow Y$ be proper surjective morphisms of complex varieties.

Definition 3. Let $I^{*}$ be any analytic subspace of $\operatorname{Isom}_{Y}^{*}\left(X, X^{\prime}\right)$. Then we say that $I^{*}$ is a quasi-meromorphic principal subspace if there exist relative quasi-meromorphic subgroups $G^{*} \cong$ Aut $X$ 粒 $X$ and $G^{* \prime} \cong$ Aut ${ }_{Y}^{*} X^{\prime}$ and a Zariski open subset $U \subseteq Y$ over which both $f$ and $f^{\prime}$ are flat, such that $I_{U}:=I^{*} \cap \operatorname{Isom}_{U}\left(X_{U}, X_{U}^{\prime}\right)$ is dense in $I^{*}$ and $I_{U} \subseteq \operatorname{Isom}_{U}\left(X_{U}, X_{U}^{\prime}\right)$ is principal with the associated relative Lie subgroups $G_{U}:=G^{*} \cap \operatorname{Aut}_{U} X_{U}$ and $G_{U}^{\prime}:=G^{* \prime} \cap \operatorname{Aut}_{U} X_{U}^{\prime}$ (cf. $\left.1.1 \mathrm{c}\right)$ ). In this case we call $G^{*}$ (resp. $G^{* \prime}$ ) associated to $I^{*}$. $I^{*}$ is called a meromorphic principal subspace if further it is proper over $Y$. In the latter case the associated $G^{*}$ and $G^{* \prime}$ are also proper over $Y$ and hence are relative meromorphic subgroups of Aut $X$ and Aut $Y X^{\prime}$ respectively.

Remark 3. 1) Let $G^{*}$ be a relative meromorphic subgroup of $\operatorname{Aut}_{Y}^{*} X$. Then the following conditions are equivalent. a) $G^{*}$ is associated to some meromorphic principal subspace $I^{*}$. b) Let $\bar{I}_{Y}^{*}\left(X, X^{\prime}\right):=\operatorname{Isom}_{Y}^{*}\left(X, X^{\prime}\right) / G^{*}$ be a relative generic quotient of $\operatorname{Isom}_{Y}^{*}\left(X, X^{\prime}\right)$ by $G^{*}$ over $Y$ with the natural projection $\varepsilon: \bar{I}_{Y}^{*}\left(X, X^{\prime}\right)$ $\rightarrow Y$ (cf. Definition 5 and Theorem 1 below). Then $\varepsilon$ admits a meromorphic section $s: Y \rightarrow I_{Y}^{*}\left(X, X^{\prime}\right)$. Moreover in this case $I^{*}$ is given by $I^{*}=\pi^{-1}(s(Y))$ and $G^{*}$ is given by the union of those irreducible components of $p_{1}(\Gamma \cap$ (Aut* $X$ $\left.\left.\times_{Y} I^{*} \times_{Y} I^{*}\right)\right)$ which are mapped surjectively onto $Y$, where $\pi: \operatorname{Isom}_{Y}^{*}\left(X, X^{\prime}\right)$ 
$\rightarrow \bar{I}_{Y}^{*}\left(X, X^{\prime}\right)$ is the natural meromorphic projection, $\Gamma \cong \operatorname{Aut}_{Y}^{*} X \times_{Y} \operatorname{Isom}_{Y}^{*}\left(X, X^{\prime}\right)$ $X_{Y} \operatorname{Isom}_{Y}^{*}\left(X, X^{\prime}\right)$ is the closure of the graph of the action of $\operatorname{Aut}_{U} X_{U}$ on $\operatorname{Isom}_{U}\left(X_{U}, X_{U}^{\prime}\right)$ and $p_{1}$ is the projection to the first factor Aut $t_{Y}^{*}$. In particular $I^{*}$ and $G^{*}$ determine each other uniquely. The analogous fact holds of course for a meromorphic subgroup $G^{* \prime} \cong$ Aut $t_{Y}^{*} X$.

c) We consider the special case of b) where $X^{\prime}=Y \times F$ for a compact complex variety $F$ and $f^{\prime}: Y \times F \rightarrow Y$ is the natural projection. Then we have the natural isomorphisms

$$
\text { Aut }_{Y} X^{\prime} \cong Y \times \text { Aut } F \text { and } \text { Aut }_{Y}^{*} X^{\prime} \cong Y \times \text { Aut* }^{*} \text {. }
$$

Definition 4. Let $I^{*}$ be a (quasi-)meromorphic principal subspace of $\operatorname{Isom}_{Y}^{*}\left(X, X^{\prime}\right)$, and $G^{*} \cong \mathrm{Aut}_{Y}^{*} X$ and $G^{* \prime} \cong \mathrm{Aut}{ }_{Y}^{*} X^{\prime}$ the associated relative (quasi-) meromorphic subgroups. Then we call $I^{*}$ admissible (with the associated meromorphic subgroup $H^{*}$ ) if $G^{* \prime}$ is of the form $G^{* \prime}=Y \times H^{*}$ for some (quasi-) meromorphic subgroup $H^{*} \subseteq$ Aut* $F$.

Suppose that $I^{*}$ is admissible as above and set $H=H^{*} \cap$ Aut $F$. Take a Zariski open subset $U \subseteq Y$ as in Definition 3. Then it is immediate to see that $f_{U}: X_{U} \rightarrow U$ is a holomorphic fiber bundle with structure group $H$. In this case we say that $f$ is a holomorphic fiber bundle over $U$ which is (quasi-)meromorphic with respect to $f$ and with (quasi-)meromorphic structure group $H$. We note that in this case the natural map $I_{U} \rightarrow U$ is the principal bundle associated to $f_{U}$.

\section{§2. Relative Generic Quotients and Related Results}

2.1. We generalize the generic quotient theorem by a meromorphic group in $[3]$ to a relative case.

Theorem 1. Let $f: X \rightarrow Y$ be a proper surjective morphism of complex varieties. Let $G^{*} \subseteq$ Aut ${ }_{Y}^{*} X$ be any relative meromorphic subgroup over $Y$. Then there exists a unique subspace $\bar{X} \subseteq D_{X / Y}{ }^{2}$ having the following properties: Let $\rho: Z \rightarrow \bar{X}$ be the universal fanily $\rho_{X / Y}: Z_{X / Y} \rightarrow D_{X / Y}$ restricted io $\bar{X}$, i.e., $Z=Z_{X / Y} \times_{D_{X / Y}} \bar{X}$. Then: 1) the natural $Y$-morphism $\pi: Z \rightarrow X$ is binneromorphic, and 2) there exists a Zariski open subset $V \subseteq \bar{X}$ such that for any $v \in V$, the corresponding subspace $Z_{v} \subseteq X_{y}$ is a closure of an orbit $Z_{v}^{0}$ of the group $G_{y}$ acting on $X_{y}$, where $y=\bar{f}(v)\left(\bar{f}: \bar{X} \rightarrow Y\right.$ biing the natural map) and $G_{y}=G_{y}^{*} \cap$ Aut $X_{y}$.

Proof. Define a meromorphic $Y$-map $\bar{\Phi}: G^{*} \times_{Y} X \rightarrow X \times_{Y} X$ by $\Phi(g, x)$ $=\left(\sigma^{*}(g, x), x\right)$. Let $R:=\Phi\left(G^{*} \times_{Y} X\right) \leqq X \times_{Y} X$. Let $p: R \rightarrow X$ be induced by the second projection $p_{2}: X \times_{Y} X \rightarrow X$;

2) the relative Douady space associated to $f$. 


$$
\begin{array}{r}
R \subseteq X \times_{Y} X \\
{ }_{p} \\
\\
X
\end{array}
$$

Let $\tau: X \rightarrow D_{X / Y}$ be the universal meromorphic $Y$-map associated to this diagram where $\tau$ is holomorphic exactly on the Zariski open subset over which $p$ is flat (cf. [2], Lemma 5.1). Let $\bar{X}$ be the image of $\tau$. Let $\bar{\tau}: X \rightarrow \bar{X}$ be the resulting meromorphic $Y$-map. We claim that this $\bar{X}$ has the desired property. Take a Zariski open subset $U \subseteq Y$ such that both $f$ and $G^{*} \rightarrow Y$ are flat over $U$, and that for each $y \in U, G_{y}$ is dense in $G_{y}^{*}$ (cf. 1.2 a)). Now we consider $\Phi$ as a meromorphic map over $X$ where $X \times_{Y} X$ is over $X$ by $p_{2}$. Take a Zariski open subset $W \leqq X$ such that for each $x \in W$, if we set $y=f(x)$, then $y \in U$, and with respect to the natural identification $\left(G^{*} \times_{Y} X\right)_{x}=G_{y}^{*}$ and $\left(X \times_{Y} X\right)_{x}=X_{y}$, $\Phi$ induces a meromorphic map $\Phi_{x}: G_{y}^{*} \rightarrow X_{y}$. Then we have for $x \in W, R_{x}=\Phi_{x}\left(G_{y}^{*}\right)$ $=\overline{\Phi_{x}\left(G_{y}\right)}=\overline{G_{y} x}$ as a subspace of $X_{y}$, where $G_{y} x$ is the orbit of $x$ under $G_{y}$ and $\overline{G_{y} x}$ its closure. In particular for any $\bar{w} \in \tau(W), Z_{\bar{w}}$ is a closure of an orbit of $G_{y}$. Since $\tau(W)$ contains a nonempty Zariski open subset, say $\left.V, 2\right)$ follows. It remains to show that $\pi$ is bimeromorphic. Restricting $W$ if necessary we may assume that $p_{W}: R_{W} \rightarrow W$ is flat [1] so that for any $x \in W$, we have $\operatorname{dim}\left(G_{f(x)} x\right)=m$ for a fixed integer $m \geqq 0$. ( $W$ is a union of 'regular orbits'. cf. [11]) Then just as in the proof of the absolute case (cf. Theorem 4.1 of [3]) we can show that $\pi$ is isomorphic on $\rho^{-1}(V) \cap \pi^{-1}(W)$. Thus $\pi$ is bimeromorphic.

It remains to show the uniqueness of $\bar{X}$. In fact, from 1) and 2) alone we deduce easily the following: 1) There exists a Zariski open subset $W_{1} \subseteq X$ such that a) for every $x \in W_{1}$ with $f(x)=y$, the point $d(x) \in D_{X / Y, y}$ corresponding to the subspace $\overline{G_{y} x}$ belongs to $\bar{X}$, and b) the set $\left\{d(x) ; x \in W_{1}\right\}$ forms a Zariski open subset of $\bar{X}$. Uniqueness clearly follows from this.

q. e. d.

Definition 5. We call the commutative diagram

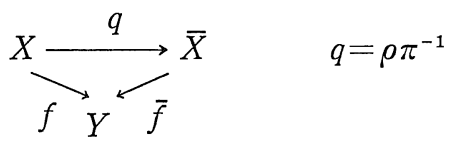

or simply, the meromorphic $Y$-map $q: X \rightarrow \bar{X}$, or $\bar{X}$ itself, the relative generic quotient of $X$ by $G^{*}$ over $Y$. We often denote $\bar{X}$ symbolically by $X / G^{*}$.

Proposition 1. Let $f: X \rightarrow Y$ and $G^{*} \subseteq$ Aut* $X$ be as in Theorem 1 .

1) Let $\tilde{Y} \rightarrow Y$ be a surjective morphism of complex varieties. Let $\tilde{X}=X \times_{Y} \tilde{Y}$ and $\tilde{G}^{*}=G^{*} \times_{Y} \tilde{Y}$. Then $\tilde{G}^{*}$ is a relative meromorphic subgroup of Aut $\tilde{X}_{Y}$ $\cong \mathrm{Aut}_{Y}^{*} X \times_{Y} \tilde{Y}$ and the relative generic quotient $\tilde{X} / \tilde{G}^{*}$ of $\tilde{X}$ by $\tilde{G}^{*}$ over $\tilde{Y}$ is isomorphic over $\tilde{Y}$ to the pull-back $\left(X / G^{*}\right) \times_{Y} \tilde{Y}$.

2) Suppose that $Y$ is a complex variety over another complex variety $T$ with a surjective morphism $h: Y \rightarrow T$. Then there exists a Zariski open subset $U \subseteq T$ 
such that for any $t \in U$ i) $G_{t}^{*}$ is a relative meromorphic subgroup of Aut ${ }_{Y_{t}} X_{t}$ over $Y_{t}$ and ii) $\left(X / G^{*}\right)_{t}=X_{t} / G_{t}^{*}$ as a subspace of $\left(D_{X / Y}\right)_{t}=D_{X_{t} / Y_{t}}$, where we consider any complex space over $Y$ naturally as a complex space over $T$ via $h$. In particular if $Y=T$ then for each $y \in U \subseteq Y,\left(X / G^{*}\right)_{y}$ is the generic quotient of $X_{y}$ by $G_{y}^{*}$. As a special case of this, if $X / G^{*} \rightarrow Y$ is bimeromorphic, then there exists a Zariski open subset $W \leqq X$ with $f(W) \leqq U$ such that if $y \in U$ then $G_{y}$ acts almost homogeneously on $X_{y}$ and its unique Zariski open orbit coincides with $W_{y}$.

3) If $G_{y}^{*}=G_{y}$ is a complex torus for $y \in U$, and $G_{y}$ acts freely on $X_{y}$ then $q_{y}: X_{y} \rightarrow X_{y} / G_{y}$ is a holomorphic fiber bundle and hence $q_{U}: X_{U} \rightarrow\left(X / G^{*}\right)_{U}$ is holomorphic and smooth.

Proof. In view of the uniqueness assertion of Theorem 1 the verification of 1) is straightforward. For the first assertion of 2) it suffices to take $U$ in such a way that for any $t \in U, \pi_{t}: Z_{t} \rightarrow X_{t}$ is bimeromorphic and $V_{t}^{-}=\bar{X}_{t}$ where $V_{t}^{-}$is the closure of $V_{t}$ in $\bar{X}_{t}$ in the notation of Theorem 1 . Here, restricting $U$ if necessary, we may assume further that $G_{U}$ is smooth over $U$. Then, when $X / G^{*} \rightarrow Y$ is bimeromorphic, if we set $A=\left\{x \in X_{U} ; \operatorname{dim} G_{U}(x)>t-r\right\} \quad\left(t=\operatorname{dim} G_{U} / U\right.$, $r=\operatorname{dim} f$ ) where $G_{U}(x)$ is the stabilizer of $G_{U, f(x)}$ at $x \in X_{f(x)}$, then $W:=X_{U}-A$ is easily seen to satisfy the above condition. In 3$)$ that $q$ is a fiber bundle is due to Holmann [10], $\S 5$. Since $\operatorname{dim} X_{\bar{x}}$ is constant on $\bar{X}_{U}$, from this follows the last assertion.

2.2. Let $f: X \rightarrow Y$ and $f^{\prime}: X^{\prime} \rightarrow Y$ be proper surjective morphism of complex varieties. Let $U \subseteq Y$ be a Zariski open subset over which both $f$ and $f^{\prime}$ are flat. Then by the universality of the relative Douady space we have the natural transformation of functors $\phi: \operatorname{Isom}_{U}\left(X_{U}, X_{U}^{\prime}\right) \rightarrow \operatorname{Isom}_{U}\left(D_{X / U}, D_{X^{\prime} / U}\right)$. Let $I^{*}$ $\subseteq$ Isom* $\left(X, X^{\prime}\right)$ be an analytic subset such that $I_{U}:=I^{*} \cap \operatorname{Isom}_{U}\left(X_{U}, X_{U}^{\prime}\right)$ is dense in $I^{*}$. Let $B \subseteq D_{X / Y}$ and $B^{\prime} \cong D_{X^{\prime} / Y}$ be analytic subspaces which are proper over $Y$ and are flat over $U$. Now we assume the following condition; $(*)$ the image of $I_{U} \cong \operatorname{Isom}_{U}\left(X_{U}, X_{U}^{\prime}\right)$ by $\phi$ is contained in the subfuctor $\mathbb{I s o m}_{U}\left(\left(D_{X_{U^{\prime}} U}, B_{U}\right)\right.$, $\left.\left(D_{X_{U} / U}, B_{U}^{\prime}\right)\right)$ (cf. 3.1 a) below for the notation) where we identify $\operatorname{Isom}_{U}\left(X_{U}, X_{U}^{\prime}\right)$ with the functor it represents. Then composed with the natural projection Isom $_{U}\left(\left(D_{X_{U} / U}, B_{U}\right),\left(D_{X_{U}^{\prime} / U}, B_{U}^{\prime}\right)\right) \rightarrow \operatorname{Isom}_{U}\left(B_{U}, B_{U}^{\prime}\right)$ we get a $U$-map $\phi: I_{U}$ $\rightarrow \operatorname{Isom}_{U}\left(B_{U}, B_{U}^{\prime}\right)$. It is immediate to see that $\phi$ is indeed a morphism of complex spaces and that $\psi$ extends to a meromorphic $Y$-map $\phi^{*}: I^{*} \rightarrow \operatorname{Isom}_{Y}^{*}\left(B, B^{\prime}\right)$. The condition (*) is fulfilled if for each $y \in U$ and for each $h \in I_{U, y} \subseteq \operatorname{Isom}\left(X_{y}, X_{y}^{\prime}\right)$ we have $D_{y} h\left(B_{y}\right)=B_{y}^{\prime}$ where $D_{y} h \in \operatorname{Isom}\left(D_{X_{y}}, D_{X_{y}^{\prime}}\right)$ is the element canonically induced by $h$.

Theorem 2. Let $I^{*} \cong \operatorname{Isom}_{Y}^{*}\left(X, X^{\prime}\right)$ be a meromorphic principal subspace with the associated relative meromorphic subgroups $G^{*} \subseteq$ Aut ${ }_{Y}^{*} X$ and $G^{* \prime} \leqq$ Aut* $X_{Y}^{*}$. Let $\bar{X}=X / G^{*}$ and $\bar{X}^{\prime}=X^{\prime} / G^{* \prime}$ be the respective relative generic quotients over $Y$. Then there exists a natural bimeromorphic $Y$-map $\bar{X} \rightarrow \bar{X}^{\prime}$ which is isomorphic 
over some Zariski open subset of $Y$.

Proof. Take Zariski open subsets $V \cong \bar{X}$ and $V^{\prime} \cong \bar{X}^{\prime}$ as in 2) of Theorem 1. Restricting $V$ and $V^{\prime}$ we may assume that the following conditions are satisfied: 1) $\bar{f}(V)=\bar{f}^{\prime}\left(V^{\prime}\right)$, and if we denote this set by $U$, then $U$ is nonsingular and Zariski open in $Y$, where $\bar{f}: \bar{X} \rightarrow Y$ and $\bar{f}^{\prime}: \bar{X}^{\prime} \rightarrow Y$ are the natural morphisms, 2) both $f$ and $f^{\prime}$ are flat over $U$, and 3) for each $y \in U$, a) $G_{y}^{*}$ is a meromorphic subgroup of Aut* $X_{y}$ and $\bar{X}_{y}$ is the generic quotient of $X_{y}$ by $G_{y}^{*}$, and the similar condition for $G_{y}^{* \prime}$ and $\bar{X}_{y}^{\prime}$ is true (cf. Proposition 1), b) $V_{y}$ is dense in $\bar{X}_{y}$ and c) the induced map $\pi_{y}: Z_{y} \rightarrow X_{y}$ is bimeromorphic where $Z$ is as in Theorem 1. Let $I_{U}=\operatorname{Isom}_{U}\left(X_{U}, X_{U}^{\prime}\right) \cap I^{*}$. Take any $y \in U$ and any $h=h_{y} \in I_{y}$ $:=I_{U, y} \leqq \operatorname{Isom}\left(X_{y}, X_{y}^{\prime}\right)$. We shall first show that $D_{y} h\left(\bar{X}_{y}\right)=\bar{X}_{y}^{\prime}$ where $D_{y} h$ is defined just before the theorem. Let $\bar{X}_{y}^{\prime \prime}=D_{y} h\left(\bar{X}_{y}\right) \leqq D_{X_{y}^{\prime}}$ and $V_{y}^{\prime \prime}=D_{y} h\left(V_{y}\right) \subseteq \bar{X}_{y}^{\prime \prime}$. Then $h$ induces an isomorphism of the following diagrams

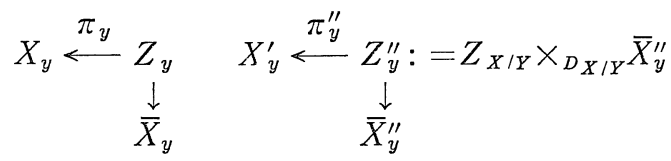

By the uniqueness of the generic quotient in Theorem 1 it suffices to show that $\bar{X}_{y}^{\prime \prime}$ satisfies the conditions of that theorem for $f_{y}^{\prime}$ and $G_{y}^{* \prime}$. Since $\pi_{y}^{\prime \prime}$ is bimeromorphic as well as $\left.\pi_{y}, 1\right)$ is satisfied. We set $G_{y}=G_{y}^{*} \cap$ Aut $X_{y}$ and $G_{y}^{\prime}=$ $G_{y}^{* \prime} \cap$ Aut $X_{y}^{\prime}$. For 2) it suffices to show that for any point $v^{\prime \prime} \in V_{y}^{\prime \prime}, Z_{v^{\prime \prime}}^{\prime \prime}$ is a closure of an orbit of $G_{y}^{\prime}$ when $Z_{v^{\prime \prime}}^{\prime \prime}$ is considered as a subspace of $X_{y}^{\prime}$ via $\pi_{y}^{\prime \prime}$. In fact, take $v \in V$ with $D_{y} h(v)=v^{\prime \prime}$. Then $Z_{v^{\prime \prime}}^{\prime \prime}=h\left(Z_{v}\right)$, which is the closure of $h\left(Z_{v}^{0}\right)$ where $Z_{v}^{0}$ is a $G_{y}$-orbit on $X_{y}$. Then, since $h_{y}$ is $\left(G_{y}, G_{y}^{\prime}\right)$-equivariant with respect to the homomorphism $h_{y_{*}}: G_{y} \rightarrow G_{y}^{\prime}$ (cf. $\left.1.1 \mathrm{c}\right)$ ), $h\left(Z_{v}^{0}\right)$ is an orbit of $G_{y}^{\prime}$ as was desired.

Thus $D_{y} h$ induces an element of Isom $\left(\bar{X}_{y}, \bar{X}_{y}^{\prime}\right)$ which we shall denote by the same letter $D_{y} h$. Hence by the remark just before the theorem we have obtained a $U$-morphism $\psi: I_{U} \rightarrow \operatorname{Isom}_{U}\left(\bar{X}_{U}, \bar{X}_{U}^{\prime}\right)$ which extends to a meromorphic $Y$-map $\psi^{*}: I^{*} \rightarrow \operatorname{Isom}_{Y}^{*}\left(\bar{X}, \bar{X}^{\prime}\right)$. Next we show that $D_{y}(h)=D_{y}\left(h^{\prime}\right)$ for any $h, h^{\prime} \in I_{y}$, $y \in U$. It suffices to show that $D_{y} h(v)=D_{y} h^{\prime}(v)$ for any $v \in V_{y}$ since $V_{y}$ is dense in $\bar{X}_{y}$. In fact, since $g\left(Z_{v}\right)=Z_{v}$ for any $g \in G_{y}$ and $h^{\prime-1} h \in G_{y}, h\left(Z_{v}\right)=h^{\prime} h^{\prime-1} h\left(Z_{v}\right)$ $=h^{\prime}\left(Z_{v}\right)$, or equivalently, $D_{y} h(v)=D_{y} h^{\prime}(v)$ as was desired. Since $I_{U} \rightarrow U$ is surjective it follows that $\psi\left(I_{U}\right) \cong \operatorname{Isom}_{U}\left(\bar{X}_{U}, \bar{X}_{U}^{\prime}\right)$ gives a holomorphic section to $\operatorname{Isom}_{U}\left(\bar{X}_{U}, \bar{X}_{U}^{\prime}\right) \rightarrow U, U$ being nonsingular, and hence, $\psi^{*}\left(I^{*}\right) \leqq \operatorname{Isom}_{Y}^{*}\left(\bar{X}, \bar{X}^{\prime}\right)$ a meromorphic section to $\operatorname{Isom}_{Y}^{*}\left(\bar{X}, \bar{X}^{\prime}\right) \rightarrow Y$. Hence by Remark 1,2$) \bar{X}$ and $\bar{X}^{\prime}$ are bimeromorphic over $Y$ by a bimeromorphic map which is isomorphic over $U$.

q. e. d.

In Theorem 2 assume that there exists a $Y$-isomorphism $\phi: X^{\prime} \rightarrow Y \times F$ for some compact complex variety $F$. Let $H^{*} \cong$ Aut* $F$ be a meromorphic subgroup. Then we say that $\psi$ is admissible with respect to $\left(I^{*}, H^{*}\right)$ if $\phi$ induces an 
isomorphism $G^{* \prime} \cong Y \times H^{*}$. Then, if $\psi$ and $\psi^{\prime}$ are $Y$-isomorphisms $X^{\prime} \cong Y \times F$ which are admissible with respect to $\left(I^{*}, H^{*}\right)$, then $\phi^{\prime} \phi^{-1}$ induces a $Y$-automorphism of $p_{1}: Y \times F \rightarrow Y$, i.e., gives a holomorphic map $Y \rightarrow$ Aut $F$, whose image is contained in $H$ where $H=H^{*} \cap$ Aut $F$. This implies that the set of admissible $Y$-isomorphisms is naturally a principal homogeneous space under the group $\mathrm{Hol}(Y, H)$, the space of holomorphic maps of $Y$ to $H$. From this observation we get the following:

Lemma 1. Suppose that there exists a $Y$-isomorphism $\phi: X^{\prime} \rightarrow Y \times F$ which is admissible with respect to $\left(I^{*}, H^{*}\right)$, so that we have the natural isomorphism $X^{\prime} / G^{* \prime} \cong Y \times\left(F / H^{*}\right)$. Then the composite meromorphic map $X \rightarrow X / G^{*} \cong X^{\prime} / G^{* \prime}$ $\cong Y \times\left(F / H^{*}\right) \rightarrow F / H^{*}$ is independent of the choice of the adnissible isomorphism $\psi$.

Definition 6. We call the meromorphic map $X \rightarrow \bar{F}:=F / H^{*}$ defined in the lemma, or any meromorphic map which is bimeromorphic to it, a canonical meromorphic map associated to $f$ and to $H^{*}$.

Clearly we have $\operatorname{dim} \bar{F}=\operatorname{dim} p$ where $p: X / G^{*} \rightarrow Y$ is the natural map.

\section{§3. Examples of Relative Quasi-Meromorphic Subgroups}

3.1. Isom ${ }_{Y}^{*}\left((X, A),\left(X^{\prime}, A^{\prime}\right)\right)$ and $\operatorname{Aut}_{Y}^{*}(X, A)$. Let $f: X \rightarrow Y$ and $f^{\prime}: X^{\prime} \rightarrow Y$ be proper morphisms of complex varieties. Let $A=\left(A_{1}, \cdots, A_{m}\right)$ and $A^{\prime}=\left(A_{1}^{\prime}, \cdots, A_{m}^{\prime}\right)$ be sequences of analytic subspaces of $X$ and $X^{\prime}$ respectively.

a) Suppose first that $f$ and $f^{\prime}$ are flat and that $A_{\alpha}^{\prime}$ are all flat over $Y$ with respect to $f^{\prime}$. Then we define a subfunctor $\operatorname{Isom}_{Y}\left((X, A),\left(X^{\prime}, A^{\prime}\right)\right):(\operatorname{An} / Y) \rightarrow$ (Sets) of $\operatorname{Isom}_{Y}\left(X, X^{\prime}\right)$ as follows ; $\operatorname{Isom}_{Y}\left((X, A),\left(X^{\prime}, A^{\prime}\right)\right)(\tilde{Y})=\left\{\varphi \in \operatorname{Isom}_{Y}\left(X, X^{\prime}\right)(\tilde{Y})\right.$; $\varphi$ induces isomorphisms of $A_{\alpha} \times_{Y} \tilde{Y}$ and $A_{a}^{\prime} \times_{Y} \tilde{Y}$ for all $\left.\alpha\right\}$.

Lemma 2. $\operatorname{Isom}_{Y}\left((X, A),\left(X^{\prime}, A^{\prime}\right)\right)$ is represented by a unique analytic subspace $\operatorname{Isom}_{Y}\left((X, A),\left(X^{\prime}, A^{\prime}\right)\right)$ of $\operatorname{Isom}_{Y}\left(X, X^{\prime}\right)$.

Proof. Let $I=\operatorname{Isom}_{Y}\left(X, X^{\prime}\right)$ and $\xi: X \times_{Y} I \rightarrow X^{\prime} \times_{Y} I$ the universal $I$-isomorphism. Let $\bar{A}_{\alpha, I}:=\xi\left(A_{\alpha} \times_{Y} I\right)$. Then by [12] Prop. 1, there exists a unique analytic subspace $T \leqq I$ such that for any morphism $u: T^{\prime} \rightarrow I$ of complex spaces $\bar{A}_{\alpha, I} \times{ }_{I} T^{\prime}=A_{\alpha, I}^{\prime} \times{ }_{I} T^{\prime}$, where $A_{\alpha, I}^{\prime}:=A_{a}^{\prime} \times_{Y} I$, as a subspace of $X_{I}^{\prime} \times{ }_{I} T^{\prime}$ if and only if $u$ factors through $T$. (In fact, apply [12] Prop. 1 to the morphism $X^{\prime} \times_{Y} I \rightarrow I$ and to the coherent analytic sheaves $\mathcal{E}:=\mathcal{O}_{A_{\alpha, I}^{\prime}}$ and $\mathscr{I}:=\mathcal{O}_{A_{a, I}^{\prime} \cap \bar{A}_{a, I}}$.) Then it is easy to see that $T$ represents the functor $\operatorname{Isom}_{Y}\left((X, A),\left(X^{\prime}, A^{\prime}\right)\right)$.

We then set $\operatorname{Aut}_{Y}(X, A)=\operatorname{Isom}_{Y}((X, A),(X, A)) . \operatorname{Aut}_{Y}(X, A)$ is a relative complex Lie subgroup of $\operatorname{Aut}_{Y} X$ over $Y$.

b) In the general case, let $U \subseteq Y$ be a Zariski open subset such that $X, X^{\prime}$, and $A_{a}^{\prime}$ are all flat over $U[1]$. 
Lemma 3. The closure $I^{-}$of $\operatorname{Isom}_{U}\left(\left(X_{U}, A_{U}\right),\left(X_{U}^{\prime}, A_{U}^{\prime}\right)\right)$ in $D_{X_{\times Y} X / Y}$ is analytic, where $A_{U}=\left(A_{1, U}, \cdots, A_{m, U}\right)$ and $A_{U}^{\prime}=\left(A_{1, U}^{\prime}, \cdots, A_{m, U}^{\prime}\right)$.

Proof. Take a proper modification $\sigma: \tilde{Y} \rightarrow Y$ such that $\sigma$ gives an isomorphism of $\sigma^{-1}(U)$ and $U$ and that the strict transforms $\tilde{X}$ and $\tilde{A}_{\alpha}$ (resp. $\tilde{X}^{\prime}$ and $\tilde{A}_{\alpha}^{\prime}$ ) of $X$ and $A_{\alpha}$ in $X \times_{Y} \tilde{Y}$ (resp. of $X^{\prime}$ and $A_{\alpha}^{\prime}$ in $X^{\prime} \times_{Y} \tilde{Y}$ ) respectively are all flat over $\tilde{Y}[9]$. Then by Lemma $2 \tilde{I}=\operatorname{Isom}_{\tilde{Y}}\left((\tilde{X}, \tilde{A}),\left(\tilde{X}^{\prime}, \tilde{A}^{\prime}\right)\right), \tilde{A}=\left(\tilde{A}_{1}, \cdots, \tilde{A}_{m}\right)$, $\tilde{A}^{\prime}=\left(\tilde{A}_{1}^{\prime}, \cdots, \tilde{A}_{m}^{\prime}\right)$, is realized as an analytic subspace of $\operatorname{Isom}_{\tilde{Y}}\left(\tilde{X}, \tilde{X}^{\prime}\right)$. Let $\hat{I}$ be the union of those irreducible components of $\tilde{I}$ whose images in $\tilde{Y}$ intersect with $\sigma^{-1}(U)$. Then the image of $\hat{I}$ in $D_{X{ }^{\prime} X^{\prime} / Y}$ by the natural proper morphism $\hat{I} \cong D_{\widetilde{X} \times \tilde{Y} \tilde{X}^{\prime} / \tilde{Y}} \cong D_{X \times X^{X^{\prime} / Y}} \times_{Y} \tilde{Y} \rightarrow D_{X \times Y^{X^{\prime}} / Y}$ is nothing but $I^{-}$.

Definition 7. $\operatorname{Isom}_{Y}^{*}\left((X, A),\left(X^{\prime}, A^{\prime}\right)\right)$ is the essential closure of $\operatorname{Isom}_{U}\left(\left(X_{U}, A_{U}\right)\right.$,

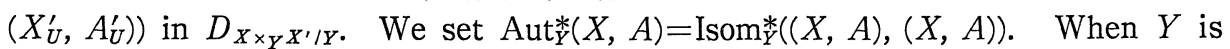
a point, we write $\operatorname{Aut}^{*}(X, A)$ for $\operatorname{Aut}_{Y}^{*}(X, A)$.

Remark 4. Aut ${ }_{Y}^{*}(X, A)$ is a relative quasi-meromorphic subgroup of Aut $X$, and $I^{*}=\operatorname{Isom}_{Y}^{*}\left((X, A),\left(X^{\prime}, A^{\prime}\right)\right)$ is a quasi-meromorphic principal subspace with the associated quasi-meromorphic subgroups $\operatorname{Aut}_{Y}^{*}(X, A)$ and $\operatorname{Aut}_{Y}^{*}\left(X^{\prime}, A^{\prime}\right)$. This follows immediately from the definitions.

c) In b) assume further that $X^{\prime}$ is of form $X^{\prime}=Y \times F$ for some compact complex variety $F$ and $f^{\prime}: X^{\prime} \rightarrow Y$ is the natural projection as in $1.3 \mathrm{c}$ ). Suppose that there exists a sequence $B=\left(B_{1}, \cdots, B_{m}\right)$ of subspaces of $F$ such that $A_{\alpha}^{\prime}=Y \times B_{\alpha} \cong X^{\prime}$. Then $\operatorname{Aut}_{Y}^{*}\left(X^{\prime}, A^{\prime}\right) \cong Y \times \operatorname{Aut}^{*}(F, B)$. Thus $\operatorname{Isom}_{Y}^{*}\left((X, A),\left(X^{\prime}, A^{\prime}\right)\right)$ is admissible, if it is not empty (Definition 4). In general, let $I^{*} \subseteq \operatorname{Isom}_{Y}^{*}\left(X, X^{\prime}\right)$ be a meromorphic principal subspace. Suppose that $I^{*}$ is admissible with the associated meromorphic subgroup $H^{*} \leqq$ Aut* $F$ and that $I^{*} \cong \operatorname{Isom}_{Y}^{*}\left((X, A),\left(X^{\prime}, A^{\prime}\right)\right)$. Then $f_{X, A}:(X, A) \rightarrow Y$ is a holomorphic fiber bundle over $U$ in the sense that for each $y \in U$ there exist a neighborhood $y \in V$ and a trivialization $X_{V} \cong V \times F$ which sends $A_{\alpha}$ onto $V \times B_{\alpha}$ isomorphically. In this case we say that $f_{X, A}$ is a holomorphic fiber bundle over $U$ which is meromorphic with respect to $f$ (and with meromorphic structure group $H$ ).

3.2. $\operatorname{Isom}_{Y}^{*}\left(X, X^{\prime}\right)_{\omega, \omega^{\prime}}$ and $\operatorname{Aut}_{Y}^{*} X_{\omega}$.

a) Let $f: X \rightarrow Y$ and $f^{\prime}: X^{\prime} \rightarrow Y$ be proper smooth morphisms of complex varieties. Let $\omega \in \Gamma\left(Y, R^{2} f_{*} \boldsymbol{R}\right)$ and $\omega^{\prime} \in \Gamma\left(Y, R^{2} f_{*}^{\prime} \boldsymbol{R}\right)$ be fixed elements. Then we define a subfunctor $\operatorname{Isom}_{Y}\left(X, X^{\prime}\right)_{\omega, \omega^{\prime}}$ of $\operatorname{Isom}_{Y}\left(X, X^{\prime}\right)$ as follows, $\operatorname{Isom}_{Y}\left(X, X^{\prime}\right)_{\omega, \omega^{\prime}}(\tilde{Y})=\left\{\varphi \in \operatorname{Isom}_{Y}\left(X, X^{\prime}\right)(\tilde{Y}) ; \varphi^{*} \omega_{\tilde{Y}}^{\prime}=\omega_{\tilde{Y}}\right\}$ where $\omega_{\tilde{Y}}$ (resp. $\omega_{\tilde{Y}}^{\prime}$ ) is the pull-back of $\omega$ (resp. $\left.\omega^{\prime}\right)$ to $X \times_{Y} \tilde{Y}$ (resp. $X^{\prime} \times_{Y} \tilde{Y}$ ).

Lemma 4. $\operatorname{Isom}_{Y}\left(X, X^{\prime}\right)_{\omega, \omega^{\prime}}$ is represented by a unique analytic subspace $\operatorname{Isom}_{Y}\left(X, X^{\prime}\right)_{\omega, \omega^{\prime}}$ of $I=\operatorname{Isom}_{Y}\left(X, X^{\prime}\right)$ which is a union of connected components.

Proof. Let $\xi: X \times_{Y} I \rightarrow X^{\prime} \times_{Y} I$ be the universal $I$-isomorphism. Let $y \in Y$ be any point and $I_{y, \gamma}$ be any connected component of $I_{y}$. For $t \in I_{y}$ let $\xi_{t}: X_{y} \rightarrow X_{y}^{\prime}$ 
be the isomorphism induced by $\xi$. Then if $\xi_{t_{0}}^{*} \omega_{I, t_{0}}^{\prime}=\omega_{I, t_{0}}$ for some $t_{0} \in I_{y, r}$, then $\xi_{t}^{*} \omega_{I, t}^{\prime}=\omega_{I, t}$ for all $t \in I_{y, r}$. From this the assertion follows readily.

We set $\operatorname{Aut}_{Y} X_{\omega}=\operatorname{Isom}_{Y}(X, X)_{\omega, \omega}$.

b) In general let $g: Z \rightarrow Y$ be any proper smooth morphism of complex varieties. Then any real closed $C^{\infty} 2$-form $\alpha$ on $Z$ determines a unique section $\bar{\alpha} \in \Gamma\left(Y, R^{2} g_{*} \boldsymbol{R}\right)$ such that the class of $\alpha_{y}$ equals $\bar{\alpha}_{y}$ in $H^{2}\left(Z_{y}, \boldsymbol{R}\right)$.

Proposition 2. Let $f, f^{\prime}, \omega, \omega^{\prime}$ be as in a). Suppose that there exists a real closed $C^{\infty}$ 2-form $\beta$ (resp. $\left.\beta^{\prime}\right)$ on $X\left(\right.$ resp. $\left.X^{\prime}\right)$ with $\bar{\beta}=\omega\left(\right.$ resp. $\left.\bar{\beta}^{\prime}=\omega^{\prime}\right)$, which restricts to a Kähler form on each fiber of $f$ (resp. $\left.f^{\prime}\right)$. Then the closure $\bar{I}$ of $\operatorname{Isom}_{Y}\left(X, X^{\prime}\right)_{\omega, \omega^{\prime}}$ in $D_{X \times X^{X^{\prime}} / Y}$ is proper over $Y$.

For the proof we need a general result. Let $f: X \rightarrow Y$ be a smooth morphism of complex varieties and $\beta$ a $C^{\infty} 2$-form on $X$ which restricts to a positive $(1,1)$ form on each fiber of $f$. Let $D_{X / Y}$ be the relative Douady space of $X$ over $Y$ and $A \subseteq D_{X / Y}$ an analytic subset. Let $\delta: A \rightarrow Y$ be the natural morphism. Then we say that $A$ is bounded with respect to $\beta$ if there exist a dense Zariski open subset $V \cong A$, a positive constant $R$ and an integer $q \geqq 0$ such that for any $d \in V$ the corresponding subspace $Z_{d} \subseteq X_{\hat{o}(d)}$ is reduced and is of pure dimension $q$ and that if $\operatorname{vol}\left(Z_{d}\right):=\int_{Z_{d}} \beta_{\delta(d)}^{q}$ is the volume of $Z_{d}$ with respect to $\beta_{\delta(d)}$ (the restriction of $\beta$ to $\left.X_{\delta(d)}\right)$, then $\operatorname{vol}\left(Z_{d}\right) \leqq R$.

Proposition 3. Let $A \subseteq D_{X / Y}$ be as above. Suppose that for any relatively compact open subset $U \leqq Y$, the restriction $A_{U}=A \cap D_{X_{U} / U}$ of $A$ over $U$ is bounded with respect to $\beta_{U}$. Then $A$ is proper over $Y$.

Proof. Follows immediately from Propositions 4.1 and 3.4 of [2]. (The proof there clearly applies also to $\beta$ as above.)

Proof of Proposition 2. In view of a) it is clear that $\bar{I}$ is a union of irreducible components of $D_{X_{\times_{Y} X^{\prime} / Y}}$. To show the properness we shall apply Proposition 3 to $A=\bar{I}$, by considering $f \times_{Y} f^{\prime}: X \times_{Y} X^{\prime} \rightarrow Y$ and $C^{\infty}$ 2-form $\tilde{\beta}_{0}:=\tilde{\beta}+\tilde{\beta}^{\prime}$ on $X \times_{Y} X^{\prime}$ instead of $f$ and $\beta$ in the proposition respectively. Here $\tilde{\beta}$ and $\tilde{\beta}^{\prime}$ are the natural pull-backs to $X \times_{Y} X^{\prime}$ of $\beta$ and $\beta^{\prime}$ respectively. Then we have to show that on any relatively compact open subset of $Y, \bar{I}$ is bounded with respect to $\tilde{\beta}_{0}$. Let $V:=\operatorname{Isom}\left(X, X^{\prime}\right)_{\omega, \omega^{\prime}} \subseteq \bar{I}$. Then for any $d \in V$ the associated subspace $Z_{d} \subseteq X_{y} \times X_{y}^{\prime}, y=\delta(d)$, equals the graph $\Gamma_{h}$ of the isomorphism $h=h_{d}: X_{y} \rightarrow X_{y}^{\prime}$ corresponding to $d$, where $\delta: I \rightarrow Y$ is the natural morphism. Hence $Z_{d} \cong X_{y}$. Moreover, since $h_{d}^{*} \omega_{y}^{\prime}=\omega_{y}$ we calculate easily that

$$
\operatorname{vol}\left(Z_{d}\right)=\int_{Z_{d}} \tilde{\beta}_{0, y}^{q}=(q+1) \int_{X_{y}} \beta_{y}^{q}
$$

where $q=\operatorname{dim} X_{y}$ (cf. the proof of Theorem 4.8 in [3]). Thus vol $\left(Z_{d}\right)$ depends only on $y=\delta(d)$ and is a continuous function of $y$. Hence it is bounded on any 
relatively compact open subset of $Y$ as was desired.

q. e. d.

c) In general let $g: Z \rightarrow Y$ be a proper morphism of complex varieties. Then we call $\alpha \in \Gamma\left(Y, R^{2} f_{*} R\right)$ a relative Kähler class if the restriction $\alpha_{y} \in H^{2}\left(X_{y}, \boldsymbol{R}\right)$ of $\alpha_{y}$ to each $X_{y}$ is a Kähler class, i. e., represented by a Kähler form. Using Proposition 2 we have shown in [6] the following:

Proposition 4. Let $f, f^{\prime}, \omega, \omega^{\prime}$ be as in a). Suppose that $\omega$ and $\omega^{\prime}$ are relative Kähler classes. Then $I$ is proper over $Y$.

Proof. See [6], Proposition 4.

d) Let $f: X \rightarrow Y$ and $f^{\prime}: X^{\prime} \rightarrow Y$ be generically smooth proper morphisms of complex varieties. Let $U \subseteq Y$ be a Zariski open subset over which both $f$ and $f^{\prime}$ are smooth. Let $\omega \in \Gamma\left(Y, R^{2} f_{*} R\right)$ and $\omega^{\prime} \in \Gamma\left(Y, R_{*}^{2} f^{\prime} R\right)$ be fixed elements.

Definition 8. $\operatorname{Isom}_{Y}^{*}\left(X, X^{\prime}\right)_{\omega, \omega^{\prime}}$ is the essential closure of $\operatorname{Isom}_{U}\left(X_{U}, X_{U}^{\prime}\right)_{\omega_{U}, \omega_{U}^{\prime}}$ in $\operatorname{Isom}_{Y}^{*}\left(X, X^{\prime}\right)$. We set Aut* $X_{\omega}=\operatorname{Isom}_{Y}^{*}(X, X)_{\omega, \omega}$.

Remark 5. $\operatorname{Isom}_{Y}^{*}\left(X, X^{\prime}\right)_{\omega, \omega^{\prime}}$ and Aut $X_{Y}^{*} X_{\omega}$ are unions of irreducible components of $\operatorname{Isom}_{Y}^{*}\left(X, X^{\prime}\right)$ and Aut $X$ respectively (cf. Lemma 4).

Proposition 5. Suppose that $\omega_{U}, \omega_{U}^{\prime}$ are relative Kähler classes, and that $f, f^{\prime} \in \mathcal{C} / Y$. Then $\operatorname{Isom}_{Y}^{*}\left(X, X^{\prime}\right)_{\omega, \omega^{\prime}}$ is proper over $Y$. Thus Aut $X_{\omega}$ and Aut $X_{\omega^{\prime}}^{\prime}$ are meromorphic subgroups of Aut $X$ and Aut $X_{Y}^{*} X^{\prime}$ respectively and $\operatorname{Isom}_{Y}^{*}\left(X, X^{\prime}\right)_{\omega, \omega^{\prime}}$ is a meromorphic principal subspace with the associated meromorphic subgroups Aut $X_{\omega}$ and Aut $_{Y}^{*} X_{\omega^{\prime}}^{\prime}$.

Proof. By Proposition $4 \operatorname{Isom}_{U}\left(X_{U}, X_{U}^{\prime}\right)_{\omega_{U}, \omega_{U}}$ has only finitely many irreducible components, say $I_{1, U}, \cdots, I_{k, U}$, which are mapped surjectively onto $U$. Then $\operatorname{Isom}_{Y}^{*}(X, X)_{\omega, \omega^{\prime}}$ is the union of the closures $I_{j}$ of $I_{j, U}$. Since $f, f^{\prime} \in \mathcal{C} / Y$, $f \times_{Y} f^{\prime} \in \mathcal{C} / Y$, and hence each $I_{j}$ are proper over $Y$ by [4]. Thus the first assertion follows. The second assertion then follows readily from the definition of these spaces.

3.3. a) Let $f: X \rightarrow Y, f^{\prime}: X^{\prime} \rightarrow Y, U \subseteq Y, \omega$ and $\omega^{\prime}$ be as in Proposition 5 . Let $A=\left(A_{1}, \cdots, A_{m}\right), A^{\prime}=\left(A_{1}^{\prime}, \cdots, A_{m}^{\prime}\right)$ be as in 3.1.

Definition 9. We set

$$
\operatorname{Isom}_{Y}^{*}\left((X, A),\left(X^{\prime}, A^{\prime}\right)\right)_{\omega, \omega^{\prime}}:=\operatorname{Isom}_{Y}^{*}\left(X, X^{\prime}\right)_{\omega, \omega^{\prime}} \cap \operatorname{Isom}_{Y}^{*}\left((X, A),\left(X^{\prime}, A^{\prime}\right)\right)
$$

and

$$
\operatorname{Aut}_{Y}^{*}(X, A)_{\omega}:=\operatorname{Aut}_{Y}^{*} X_{\omega} \cap \operatorname{Aut}_{Y}^{*}(X, A) 。
$$

Remark 6. 1) $\operatorname{Isom}_{Y}^{*}\left((X, A),\left(X^{\prime}, A^{\prime}\right)\right)_{\omega, \omega^{\prime}}$ is a meromorphic principal subspace with the associated meromorphic subgroups $\operatorname{Aut}_{Y}^{*}(X, A)_{\omega}$ and $\operatorname{Aut}_{Y}^{*}\left(X^{\prime}, A^{\prime}\right)_{\omega^{\prime}}$.

2) There exists a Zariski open subset $U \subseteq Y$ such that

$$
\left(\operatorname{Isom}_{Y}^{*}\left((X, A),\left(X^{\prime}, A^{\prime}\right)\right)_{\omega, \omega^{\prime}}\right)_{y}=\operatorname{Isom} *\left(\left(X_{y}, A_{y}\right),\left(X_{y}^{\prime}, A_{y}\right)\right)_{\omega_{y}, \omega_{y}^{\prime}}
$$


for any $y \in U$.

3) Let $\nu: \tilde{Y} \rightarrow Y$ be a surjective morphism of complex varieties. Let $\tilde{X}=X \times_{Y} \tilde{Y}$ and $\tilde{A}=\left(A_{1} \times_{Y} \tilde{Y}, \cdots, A_{m} \times_{Y} \tilde{Y}\right)$. Let $\tilde{\omega}$ be the pull-back of $\omega$ to $\tilde{X}$. Then $\operatorname{Aut}_{Y}^{*}(X, A)_{\omega} \times_{Y} \tilde{Y} \cong \operatorname{Aut}_{\tilde{Y}}^{*}(\tilde{X}, \tilde{A})_{\varpi}$ with respect to the natural isomorphism $\operatorname{Aut}^{*} X \times_{Y} \tilde{Y} \cong \operatorname{Aut}_{\tilde{Y}}^{*} \tilde{X}$.

In fact, since Isom ${ }_{Y}^{*}\left(X, X^{\prime}\right)_{\omega, \omega^{\prime}}$ is a union of irreducible components (Remark 5) it follows that $\operatorname{Isom}_{Y}^{*}\left((X, A),\left(X^{\prime}, A^{\prime}\right)\right)_{\omega, \omega^{\prime}}$ is the essential closure of $\operatorname{Isom}_{U}\left(X_{U}, X_{U}^{\prime}\right) \omega_{U}, \omega_{U}^{\prime} \cap \operatorname{Isom}_{U}\left(\left(X_{U}, A_{U}\right),\left(X_{U}^{\prime}, A_{U}^{\prime}\right)\right)$. From this together with Remark 4 and Proposition 5, 1) follows. 2) is standard (cf. 1.2 a)). For 3) it suffices to see that $\operatorname{Aut}_{Y}^{*}(X, A) \times_{Y} \tilde{Y} \cong \operatorname{Aut}_{\tilde{Y}}^{*}(\tilde{X}, \tilde{A})$ and $\left(\operatorname{Aut}_{Y}^{*} X_{\omega}\right) \times_{Y} \tilde{Y} \cong \operatorname{Aut}_{\tilde{Y}}^{*} \tilde{X}_{\varpi}$. Since $\nu$ is surjective, this follows from the isomorphisms $\operatorname{Aut}_{U}\left(X_{U}, A_{U}\right) \times_{U} \widetilde{U} \cong \operatorname{Aut}_{\widetilde{U}}\left(\tilde{X}_{\widetilde{U}}, \tilde{A}_{\widetilde{U}}\right)$ and $\left(\operatorname{Aut}_{U} X_{U}\right) \omega_{U} \times{ }_{U} \tilde{U} \cong\left(\operatorname{Aut}_{\tilde{U}} X_{\widetilde{U}}\right)_{\tilde{U}}$ where $\tilde{U}=\nu^{-1}(U)$.

b) Consider the special case where $X^{\prime}=Y \times F$ for some compact complex variety $F$ and $f^{\prime}: X^{\prime} \rightarrow Y$ is the natural projection. Let $B=\left(B_{1}, \cdots, B_{m}\right)$ be a sequence of subspaces of $F$ as in $3.1 \mathrm{c}$ ). Suppose that $\omega^{\prime}$ is of the form $\omega^{\prime}=p^{*} \omega_{0}$ for some Kähler class $\omega_{0}$ on $F$ where $p: X^{\prime} \rightarrow F$ is the natural projection. Then:

Proposition 6. If $\operatorname{Isom}_{Y}^{*}\left((X, A),\left(X^{\prime}, A^{\prime}\right)\right)_{\omega, \omega^{\prime}} \neq \varnothing$, then $f_{X, A}$ is a holomorphic fiber bundle over $U$ which is meromorphic with respect to $f$ and with meromorphic structure group $\operatorname{Aut}(F, B)_{\omega_{0}}$ in the sence of $\left.3.1 \mathrm{c}\right)$.

Proof. We have $\operatorname{Aut}_{Y}^{*}\left(X^{\prime}, A^{\prime}\right)_{\omega^{\prime}} \cong Y \times \operatorname{Aut}^{*}(F, B)_{\omega_{0}}$ and hence $\operatorname{Isom}_{Y}^{*}((X, A)$, $\left.\left(X^{\prime}, A^{\prime}\right)\right)_{\omega, \omega^{\prime}}$ is admissible. Thus the proposition follows from $\left.3.1 \mathrm{c}\right)$.

3.4. Let $f: X \rightarrow Y$ be a proper flat morphism of complex varieties. Let Aut $_{Y, 0} X$ be the unique irreducible component of Aut $_{Y} X$ which contains the identity section $e(Y)$. Then it is easy to see that $\operatorname{Aut}_{Y, 0} X$ is a relative complex Lie subgroup of $\operatorname{Aut}_{Y} X$.

Lemma 5. Suppose that $f \in \mathcal{C} / Y$. Then there exists a Zariski open subset $U \subseteq Y$ such that $\left(\operatorname{Aut}_{Y, 0} X\right)_{y}=\operatorname{Aut}_{0} X_{y}$ for each $y \in U$ where Aut $_{0} X_{y}$ is the identity component of Aut $X_{y}$.

Proof. Let $\mu:$ Aut $_{Y, 0} X \rightarrow Y$ be the natural morphism. Let $r=\operatorname{dim} \mu$, and $V=\left\{y \in Y ; \operatorname{dim}_{e(y)} \mu^{-1}(y)=r\right.$, and $Y$ is smooth at $\left.y\right\}$. Then $V$ is Zariski open in $Y$. Moreover $\mu$ is smooth at every point of $e(V)$ and hence $\operatorname{Aut}_{Y, 0} X$ is smooth along $e(V)$. Let $A=\operatorname{Aut}_{Y, 0} X$ and $n: \tilde{A} \rightarrow A$ the normalization. Since $n$ is isomorphic along $e(V), e$ lifts to a meromorphic section $\tilde{e}$ to $\tilde{\mu}: \tilde{A} \rightarrow Y$. On the other hand, since $f \in \mathcal{C} / Y, \tilde{\mu}$ is proper [4]. Let $b: \tilde{A} \rightarrow \tilde{Y}, c: \tilde{Y} \rightarrow Y$ be the Stein factorization of $\tilde{\mu}$. Then $b \tilde{e}$ gives a meromorphic section to c. Hence the fiber of $\tilde{\mu}$ is connected. Since $\tilde{A}$ is normal, this implies that the general fiber of $\tilde{\mu}$, and hence of $\mu$, is irreducible. Thus for general $y \in Y, A_{y}$ is the closure of Aut $_{0} X_{y}$. Hence the assertion follows.

Let $f: X \rightarrow Y$ be a proper surjective morphism of complex varieties. Let 
$U \subseteq Y$ be a Zariski open subset over which $f$ is smooth. Then we denote by Aut ${ }_{Y, 0} X$ the closure of Aut $_{U, 0} X_{U}$ in Aut $X$. This is independent of the choice of $U$ as above. Aut ${ }^{*}, 0 X$ is a relative meromorphic subgroup if $f \in C / Y$.

Proposition 7. Let $f: X \rightarrow Y$ be a proper morphism of complex spaces. Let $U \subseteq Y$ be a Zariski open subset. 1) Suppose that $f$ is smooth over $U$ with each fiber a complex torus and that $f$ admits a holomorphic section $e_{U}: U \rightarrow X_{U}$ on $U$. Then $f_{U}: X_{U} \rightarrow U$ has the unique structure of a complex Lie group over $U$ with $e_{U}$ the identity section. 2) Suppose further that $X, Y$ are varieties, $f \in \mathcal{C} / Y$ and that $e_{U}$ extends to a meromorphic section $e^{*}: Y \rightarrow X$. Then the group law of $X_{U}$ over $U$ extends meromorphically over $Y$.

Proof. 1) Restricting the natural relative action $\sigma_{U}$ : $\left(\right.$ Aut $\left._{U, 0} X_{U}\right) \times_{U} X_{U} \rightarrow X_{U}$ to $\left(\operatorname{Aut}_{U, 0} X_{U}\right) \times_{U} e_{U}(U) \cong \mathrm{Aut}_{U, 0} X_{U}$ we get an isomorphism $\eta_{U}: \mathrm{Aut}_{U, 0} X_{U} \cong X_{U}$ (cf. Appendix). Hence 1) follows. (For the uniqueness see [11], Cor. 6.6.) 2) Similarly, restricting $\sigma^{*}:$ Aut ${ }_{Y, 0}^{*} X \times_{Y} X \rightarrow X$ to Aut ${ }_{Y, 0} X \times_{Y} e(Y)$, which is bimeromorphic to Aut ${ }_{Y, 0} X$ we get a natural bimeromorphic map Aut ${ }_{Y, 0}^{*} X \rightarrow X$ extending $\eta_{U}$. Then 2) follows from Remark 1,3).

q. e. d.

3.5. In concluding this section, as an application of Theorem 2 combined with the consideration of this section, we shall prove a proposition which is used in [5].

Let $g: X \rightarrow Y, h: Y \rightarrow T$ be fiber spaces $^{3)}$ of complex varieties. Let $A=\left(A_{1}, \cdots, A_{m}\right)$ be a sequence of analytic subspaces of $X$. Suppose that 1) there exist Zariski open subsets $U \subseteq T, V \cong Y$ with $h(V) \cong U$ such that for any $u \in U, g_{u}=g_{u, X_{u}, A_{u}}:\left(X_{u}, A_{u}\right) \rightarrow Y_{u}$ is a holomorphic fiber bundle over $V_{u} \subseteq Y_{u}$ which is meromorphic with respect to $g_{u}$ (cf. 3.1, c)) and 2) there exists a holomorphic section $s: T \rightarrow Y$ with $s(T) \cap V \neq \varnothing$. Suppose further that $g$ is Kähler (cf. [4]) so that in particular we can find a relative Kähler class $\omega \in \Gamma\left(Y, R^{2} g_{*} \boldsymbol{R}\right)$ over $Y$. Then by Proposition 6 if $s(u) \in V$ we can take $G^{*}(u):=\operatorname{Aut} *\left(X_{s(u)}\right.$, $\left.A_{s(u)}\right)_{\omega_{s}(u)}$ as a meromorphic structure group of $g_{u}$ (considering $\left(X_{s(u)}, A_{s(u)}\right)$ as a typical fiber of the bundle). Then we shall prove the following:

Proposition 8. Under the above situation there exists a commutative diagram

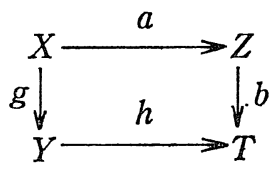

where $a$ is a surjective meromorphic map and $b$ is a fiber space of complex varieties, such that if we restrict $U$ smaller, then for each $u \in U, Z_{u}$ is a generic quotient $X_{u} / G^{*}(u)$ of $X_{u}$ by $G^{*}(u)$ and $a$ induces a canonical meromorphic map $a_{u}: X_{u} \rightarrow Z_{u}$ associated to $g_{u}$ and $G^{*}(u)$ (cf. Def. 6).

3) A fiber space is a proper surjective morphism with general fiber irreducible. 
Proof. Let $\hat{X}:=X \times_{Y} T$ where $T$ is over $Y$ via s. Let $X^{\prime}:=\hat{X} \times{ }_{T} Y$ and $g^{\prime}: X^{\prime} \rightarrow Y$ the natural map. Let $\hat{\omega}$ (resp. $\omega^{\prime}$ ) be the pull-back of $\omega$ (resp. $\hat{\omega}$ ) to $\hat{X}$ (resp. $X^{\prime}$ ). Then $g^{\prime}$ is a Kähler morphism with a relative Kähler class $\omega^{\prime} \in \Gamma\left(Y, R^{2} g_{*}^{\prime} \boldsymbol{R}\right)$. Let $\hat{A}_{i}:=A_{i} \times_{Y} T \subseteq \hat{X} \quad$ and $\quad A_{i}^{\prime}:=\hat{A}_{i} \times_{T} Y \leqq X^{\prime}$. Let $I^{*}:=\operatorname{Isom}_{Y}^{*}\left((X, A),\left(X^{\prime}, A^{\prime}\right)\right)_{\omega, \omega^{\prime}}$ where $A:=\left(A_{1}, \cdots, A_{m}\right)$ and $A^{\prime}:=\left(A_{1}^{\prime}, \cdots, A_{m}^{\prime}\right)$. Then by Remark 6,1$) I^{*}$ is a principal meromorphic subspace to which $G^{*}$ $:=\operatorname{Aut}_{Y}^{*}(X, A)_{\omega}$ and $G^{* \prime}:=\operatorname{Aut}_{Y}^{*}\left(X^{\prime}, A^{\prime}\right)_{\omega^{\prime}}$ are associated. Let $\bar{X}=X / G^{*}$ (resp. $\bar{X}^{\prime}=X^{\prime} / G^{* \prime}$ ) be the relative generic quotient of $X$ by $G^{*}$ (resp. $X^{\prime}$ by $G^{* \prime}$ ) over $Y$. Then by Theorem 2 there exists a canonical bimeromorphic map $\eta: \bar{X} \rightarrow \bar{X}^{\prime}$ over $Y$. On the other hand, by Remark 6, 3) $G^{* \prime}=\hat{G}^{*} \times_{T} Y$ where $\hat{G}^{*}$ $:=\operatorname{Aut}_{T}^{*}(\hat{X}, \hat{A})_{\hat{\omega}}, \hat{A}=\left(\hat{A}_{1}, \cdots, \hat{A}_{m}\right)$. Further we have the natural meromorphic map $\pi: \bar{X}^{\prime} \rightarrow \hat{X} / \hat{G}^{*}$ over $T$ (cf. Proposition 1,1)). Let $Z:=\hat{X} / \hat{G}^{*}$ and define $a: X \rightarrow Z$ by the composite meromorphic map $\pi \eta q: X \rightarrow Z$ where $q: X \rightarrow \bar{X}$ is the quotient meromorphic map. Let $b: Z \rightarrow T$ be the natural surjective morphism. Then we have $h f=b a$. We claim that the resulting diagram meets the requirement of the proposition. In fact, restricting $U$ smaller, we have that for each $u \in U, G_{u}^{*}$ is a relative meromorphic subgroup of Aut ${ }_{Y_{u}} X_{u}$ over $Y_{u}$ and $\bar{X}_{u}=X_{u} / G_{u}^{*}$ (cf. Proposition 1,2$)$ ), where $X_{u} / G_{u}^{*}$ is a relative generic quotient of $X_{u}$ by $G_{u}^{*}$ over $Y_{u}$. Further we have $G_{s(u)}^{*}=G^{*}(u)$ and $Z_{u}:=\left(\hat{X} / \hat{G}^{*}\right)_{u} \cong X_{s(u)} / G^{*}(u)$. Combining these facts we see readily from our construction that for sufficiently small $U$, the induced meromorphic map $a_{u}: X_{u} \rightarrow Z_{u}$ is a canonical meromorphic map associated to $G^{*}(u)$.

q. e. d.

\section{$\S 4$. $\mathrm{BHol}_{Y}^{*}\left(X, X^{\prime}\right)$}

a) Let $f: X \rightarrow Y$ and $f^{\prime}: X^{\prime} \rightarrow Y$ be proper flat morphisms of complex varieties. Let $\operatorname{Hol}_{Y}\left(X, X^{\prime}\right)$ be the contravariant functor $(\mathrm{An} / Y) \rightarrow$ (Sets) defined by $\operatorname{Hol}_{Y}\left(X, X^{\prime}\right)(\tilde{Y}):=$ the set of $\tilde{Y}$-morphisms $\phi: X \times_{Y} \tilde{Y} \rightarrow X^{\prime} \times_{Y} \tilde{Y}$. Then $\operatorname{Hol}_{Y}\left(X, X^{\prime}\right)$ is represented by a unique Zariski open subset $\operatorname{Hol}_{Y}\left(X, X^{\prime}\right)$ of the relative Douady space $D_{X \times_{Y} X^{\prime} / Y}$ with $\operatorname{Isom}_{Y}\left(X, X^{\prime}\right) \subseteq \operatorname{Hol}_{Y}\left(X, X^{\prime}\right)$ (cf. [13]).

Suppose for simplicity that both $f$ and $f^{\prime}$ are smooth with connected fibers. Let $\operatorname{BHol}_{Y}\left(X, X^{\prime}\right):=\bigcup_{y \in Y} \operatorname{BHol}_{Y}\left(X, X^{\prime}\right)_{y}$ where $\operatorname{BHol}_{Y}\left(X, X^{\prime}\right)_{y}:=\left\{h \in \operatorname{Hol}_{Y}\left(X, X^{\prime}\right)_{y}\right.$; $h(y)$ is bimeromorphic , where $h(y): X_{y} \rightarrow X_{y}^{\prime}$ is a morphism corresponding to $h$. Then $\operatorname{BHol}_{Y}\left(X, X^{\prime}\right)$ is Zariski open in $D_{X \times_{Y} X^{\prime} / Y}$ (cf. [2], Lemma 5.5). We see that for any open subset $W \subseteq Y$ there is a natural bijective correspondence between the set of holomorphic sections of $\operatorname{BHol}_{Y}\left(X, X^{\prime}\right) \rightarrow Y$ on $W$ and the set of bimeromorphic morphisms $X \rightarrow X^{\prime}$ over $W$.

Let $A \subseteq X$ and $A^{\prime} \leqq X^{\prime}$ be any analytic subspaces. Suppose that $A^{\prime}$ is flat over $Y$. Then the subfunctor $\mathbf{H o l}_{Y}\left((X, A),\left(X^{\prime}, A^{\prime}\right)\right)$ of $\mathbf{H o l}_{Y}\left(X, X^{\prime}\right)$ defined by $\mathrm{Hol}_{Y}\left((X, A),\left(X^{\prime}, A^{\prime}\right)\right)(\tilde{Y})=\left\{\psi \in \operatorname{Hol}_{Y}\left(X, X^{\prime}\right)(\tilde{Y}) ; \psi(A)=A^{\prime}\right\}$ is represented by a unique analytic subspace $\operatorname{Hol}_{Y}\left((X, A),\left(X^{\prime}, A^{\prime}\right)\right)$ of $\operatorname{Hol}_{Y}\left(X, X^{\prime}\right)$. This can be shown just in the same way as for Lemma 2 . We set $\operatorname{BHol}_{Y}\left((X, A),\left(X^{\prime}, A^{\prime}\right)\right)$ : 
$=\operatorname{Hol}_{Y}\left((X, A),\left(X^{\prime}, A^{\prime}\right)\right) \cap \mathrm{BHol}_{Y}\left(X, X^{\prime}\right)$.

b) Let $f: X \rightarrow Y$ and $f^{\prime}: X^{\prime} \rightarrow Y$ be generically smooth proper surjective morphisms of complex varieties with connected fibers. Let $U \subseteq Y$ be a Zariski open subset over which both $f$ and $f^{\prime}$ are smooth. Then $\mathrm{BHol}_{U}\left(X_{U}, X_{U}^{\prime}\right)$ is

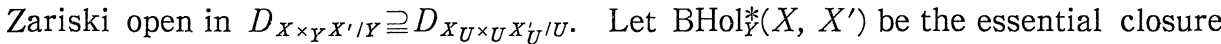
(1.2 a)) of $\mathrm{BHol}_{U}\left(X_{U}, X_{U}^{\prime}\right)$ in $D_{X_{\times} X^{X^{\prime} / Y}}$ which is independent of the choice of $U$. Let $A \subseteq X$ and $A^{\prime} \cong X$ be analytic subspaces. Restrict $U$ smaller so that $A^{\prime}$ is flat over $U$. Then the closure of $\operatorname{BHol}_{U}\left(\left(X_{U}, A_{U}\right),\left(X_{U}^{\prime}, A_{U}^{\prime}\right)\right)$ is analytic in $D_{X \times Y^{X^{\prime}} / Y}$ (cf. the proof of Lemma 3). We shall denote the essential closure of $\mathrm{BHol}_{U}\left(\left(X_{U}, A_{U}\right),\left(X_{U}^{\prime}, A_{U}^{\prime}\right)\right)$ in $D_{X \times Y^{X^{\prime} / Y}}$ by $\mathrm{BHol}_{Y}^{*}\left((X, A),\left(X^{\prime}, A^{\prime}\right)\right)$.

Remark 7. 1) A bimeromorphic morphism $\psi: X_{U} \rightarrow X_{U}^{\prime}$ defined on $U$ extends to a bimeromorphic map $\psi^{*}: X \rightarrow X^{\prime}$ over $Y$ if and only if the corresponding holomorphic section $U \rightarrow \mathrm{BHol}_{U}\left(X_{U}, X_{U}^{\prime}\right)$ extends to a meromorphic section $Y \rightarrow \mathrm{BHol}_{Y}^{*}\left(X, X^{\prime}\right)$.

2) If $\tilde{Y} \rightarrow Y$ is a surjective morphism of complex varieties, then it is immediate to see that $\operatorname{BHol}_{Y}^{*}\left(X, X^{\prime}\right) \times_{Y} \tilde{Y} \cong \mathrm{BHol}_{Y}^{*}\left(X \times_{Y} \tilde{Y}, X \times_{Y} \tilde{Y}\right)$

3) If $f \in \mathcal{C} / Y$, then after replacing $Y$ by any relatively compact open subset of $Y$ any irreducible component of $\mathrm{BHol}_{Y}^{*}\left(X, X^{\prime}\right)$ (resp. BHol $\left((X, A),\left(X^{\prime}, A^{\prime}\right)\right)$ is proper over $Y$. In particular if $X$ is compact, we need no restriction to a relatively compact subset.

c) We shall include a standard application of Remark 7,3) as a reference to $[5]$.

Let $f: X \rightarrow Y$ and $f^{\prime}: X^{\prime} \rightarrow Y$ be surjective morphisms of compact complex varieties in $\mathcal{C}$. Let $U \subseteq Y$ be a Zariski open subset over which both $f$ and $f^{\prime}$ are smooth.

Proposition 9. 1) Suppose that $f$ and $f^{\prime}$ admit meromorphic sections $s: Y \rightarrow X$ and $s^{\prime}: Y \rightarrow X^{\prime}$ respectively. Suppose further that there exists a $U$ isomorphism $\eta: X_{U} \rightarrow X_{U}^{\prime}$ with $\left.\eta s\right|_{U}=\left.s^{\prime}\right|_{U}$. Then if $\operatorname{Aut}_{0}\left(X_{u}, s(u)\right)=\{e\}$ for all $u \in U$, then $\eta$ extends to a bimeromorphic $Y$-map $\left.\eta^{*}: X \rightarrow X^{\prime} .2\right)$ Suppose that $\mathrm{BHol}\left(X_{u}, X_{u}^{\prime}\right)$ (resp. Isom $\left(X_{u}, X_{u}^{\prime}\right)$ ) are nonempty and discrete for all $u \in U$. Then there exists a finite covering $\mu: \tilde{Y} \rightarrow Y$ such that $X \times_{Y} \tilde{Y}$ and $X^{\prime} \times_{Y} \tilde{Y}$ is bimeromorphic over $\tilde{Y}$ by a bimeromorphic $\tilde{Y}$-map which is holomorphic (resp. isomorphic) over $\tilde{U}=\mu^{-1}(U)$.

Proof. 1) Let $I^{*}=\operatorname{Isom}_{Y}^{*}\left((X, s(Y)),\left(X^{\prime}, s^{\prime}(Y)\right)\right)$ and $I_{U}=\operatorname{Isom}_{U}\left(\left(X_{U}, s(U)\right)\right.$, $\left.\left(X_{U}^{\prime}, s^{\prime}(U)\right)\right)$. Then $\eta$ defines a holomorphic section $\sigma$ to $I_{U} \rightarrow U$. Let $I_{1}^{*}$ be the irreducible component of $I^{*}$ containing $\sigma(U)$. Since $I_{U, u}=\operatorname{Isom}\left(\left(X_{u}, s(u)\right),\left(X_{u}^{\prime}, s^{\prime}(u)\right)\right)$ $\cong$ Aut $\left(X_{u}, s(u)\right)$, from our assumption it follows that $I_{1}^{*}$ is discrete over $U$. Hence $I^{*} \rightarrow Y$ is generically finite so that it coincides with the closure of $\sigma(U)$. Namely, $\sigma$ extends to a meromorphic section $Y \rightarrow I^{*}$. Hence the proposition follows from Remark 1,2). 2) By our assumption we infer readily that there 
exists an irreducible component $\tilde{Y}$ of $\mathrm{BHol}_{Y}^{*}\left(X, X^{\prime}\right)$ such that $\tilde{Y} \cap \mathrm{BHol}_{U}\left(X_{U}, X_{U}^{\prime}\right)$ is dense in $\tilde{Y}$ and the natural morphism $\mu: \tilde{Y} \rightarrow Y$ is generically finite and surjective. Let $\tilde{X}=X_{\tilde{Y}}$ and $\tilde{X}^{\prime}=X_{\tilde{Y}}^{\prime}$. Since $\tilde{Y} \times_{Y} \tilde{Y} \cong \mathrm{BHol}_{Y}^{*}\left(X, X^{\prime}\right) \times_{Y} \tilde{Y}$ $\cong \operatorname{BHol}_{\tilde{Y}}^{*}\left(\tilde{X}, \tilde{X}^{\prime}\right), \operatorname{BHol}_{\tilde{Y}}^{*}\left(\tilde{X}, \tilde{X}^{\prime}\right) \rightarrow \tilde{Y}$ admits a natural holomorphic section whose image over $\tilde{U}$ is in $\operatorname{BHol}_{\tilde{U}}\left(\tilde{X}_{\widetilde{U}}, \tilde{X}_{\widetilde{U}}^{\sim}\right)$. Hence $f_{\tilde{Y}}$ and $f_{\tilde{Y}}^{\prime}$ are bimeromorphic. Let $\tilde{Y} \rightarrow \tilde{Y}_{1} \rightarrow Y$ be the Stein factorization of $\mu$. Then replacing $\tilde{Y}$ by $\tilde{Y}_{1}$ which is bimeromorphic to $\tilde{Y}$ we obtain 2). For Isom the proof is similar. q.e.d.

Remark 8. As is clear from the above proof the conclusion of 2 ) is true if there exists an analytic subset $\tilde{Y}^{\prime} \subseteq \mathrm{BHol}_{Y}^{*}\left(X, X^{\prime}\right)\left(\operatorname{resp} . \operatorname{Isom}_{Y}^{*}\left(X, X^{\prime}\right)\right)$ such that $\tilde{Y}^{\prime} \cap \mathrm{BHol}_{U}\left(X_{U}, X_{U}^{\prime}\right)$ (resp. $\tilde{Y}^{\prime} \cap \operatorname{Isom}_{U}\left(X_{U}, X_{U}^{\prime}\right)$ ) is dense in $\tilde{Y}^{\prime}$ and that $\tilde{Y}_{y}^{\prime}$, $y \in U$, is discrete. Moreover these results (Proposition 9 and this remark) are true even if the assumption is weakened to: $f, f^{\prime} \in \mathcal{C} / Y$ ( $Y$ may not be compact), except that for 2 ) we have to replace $Y$ by an arbitrary relatively compact open subset in the conclusion.

\section{Appendix}

In this appendix we shall summarize some well-known results on the automorphism group of a complex torus and its relative form.

a) Let $T$ be a complex torus and $o \in T$ a fixed point. Then $T$ has a unique structure of a complex Lie group with identify $o$. Then we can identify $T$ with Aut $_{0} T$ naturally. Let $\Gamma=H_{1}(T, Z)$ and $H(T) \subseteq$ Aut $T$ the Lie subgroup of isomorphisms of $T$ as a complex Lie group. We note that $H(T)=\operatorname{Aut}(T,\{0\})$. Then we have the exact sequence

$$
0 \longrightarrow T \longrightarrow \text { Aut } T \stackrel{\alpha}{\longrightarrow} \text { Aut } \Gamma
$$

and if $H$ is the image of $\alpha$, then $\alpha$ induces an isomorphism $H(T) \cong H$. Hence we have the natural semi-direct product decomposition Aut $T=T \cdot H(T)$.

b) Let $f: X \rightarrow Y$ be a proper smooth morphism of complex spaces (not necessarily reduced). Suppose that each fiber of $f$ is a complex torus and $f$ admits a holomorphic section $s: Y \rightarrow X$. Then $X$ has a unique structure of a relative complex Lie group over $Y$. In fact we can identify $X$ with Aut $_{Y, 0} X$ in the notation of 3.4 (cf. Proposition 7). Let $H_{Y} X$ be the relative complex Lie subgroup of Aut $_{Y} X$ defined by $H_{Y} X=\operatorname{Aut}_{Y}(X, s(Y))$. Then we have $\left(H_{Y} X\right)_{y}$ $=H\left(X_{y}\right)$ for each $y \in Y$. Let $\Gamma_{Y}$ be the local system of abelian groups on $Y$ defined by the presheaf $U \rightarrow H_{1}\left(X_{U}, \mathbb{Z}\right)$ with $U$ open subsets of $Y$. Let $r:$ Aut $_{Y} \Gamma_{Y} \rightarrow Y$ be the relative automorphism group of $\Gamma_{Y} \rightarrow Y ; r$ represents the functor $K:(\mathrm{An} / Y) \rightarrow($ Sets $)$ with $K(\tilde{Y})=$ the set of $\tilde{Y}$-automorphisms of $\Gamma \times_{Y} \tilde{Y}$. Aut $_{Y} \Gamma_{Y}$ is a relative complex Lie group over $Y$ with $r$ locally biholomorphic. Then as in the absolute case we have the exact sequence

$$
0 \longrightarrow X \longrightarrow \operatorname{Aut}_{Y} X \stackrel{\alpha_{Y}}{\longrightarrow} \operatorname{Aut}_{Y} \Gamma_{Y}
$$


of relative complex Lie groups in the sense that each map is a morphism of complex spaces over $Y$ and induces an exact sequence of complex Lie groups on each fiber. Hence $\alpha_{Y}$ induces an isomorphism of $H_{Y} X$ with a relative subgroup of $\operatorname{Aut}_{Y} \Gamma_{Y}$, and we have the semi-direct product decomposition

over $Y$.

$$
\operatorname{Aut}_{Y} X=X \cdot H_{Y} X
$$

\section{References}

[1] Frisch, J., Points de platitude d'un morphisme d'espaces analytiques complexes, Inventiones math., 4 (1967), 118-138.

[2] Fujiki, A., Closedness of the Douady spaces of compact Kähler spaces, Publ. RIMS, Kyoto Univ., 14 (1978), 1-51.

[3] —, On automorphism groups of compact Kähler manifolds, Inventiones math., 44 (1978) , 225-258.

[4] - On the Douady space of a compact complex space in the category $\mathcal{C}$, Nagoya J. Math., 85 (1982), 189-211.

[5] - On the structure of compact complex manifolds in $\mathcal{C}$, Advanced Studies in Pure Math., 1, ed. S. Iitaka and H. Morikawa, (1982), 229-300.

[6] —, Coarse moduli spaces for polarized compact Kähler manifolds and polarized algebraic manifolds, to appear.

[7] Grothendieck, A., A general theory of fiber spaces with structure sheaf, University of Kansas, 1955.

[8] - Technique de construction en géométrie analytique, Séminaire H. Cartan, 13e année (1960/61).

[9] Hironaka, H., Flattening theorem in complex analytic geometry, Amer. J. Math., 96 (1975), 503-547.

[10] Holmann, H., Quotienten Komplexer Räume, Math. Ann., 142 (1961), 407-440.

[11] Mumford, D., Geometric invariant theory, Berlin-Heidelberg-New York, Springer, 1965.

[12] Pourcin, G., Théorème de Douady audessus de S, Ann. Sci. Norm. Sup. di Pisa, 23 (1969), 451-459.

[13] Schuster, H.W., Zur Theorie der Deformationen kompakter komplexer Räume, Inventiones math., 9 (1970), 284-294. 